\title{
Nonlinearity Modeling of Chireix Outphasing Power Combiner Under Amplitude Imbalance
}

\author{
Pavel Afanasyev ${ }^{\# 1}$, Prasidh Ramabadran ${ }^{\# 2}$, Ronan Farrell ${ }^{\# 3}$, John Dooley ${ }^{\# 4}$, \\ \#National University of Ireland Maynooth, Ireland \\ ${ }^{1}$ pavel.afanasyev.2017@mumail.ie, ${ }^{2}$ Prasidh.Ramabadran@mu.ie, ${ }^{3}$ Ronan.Farrell@mu.ie, ${ }^{4}$ John.Dooley@mu.ie
}

\begin{abstract}
This work proposes a concise standalone model for Chireix power combiner. The model is based on the analysis of the nonlinear behaviour of a Chireix power combiner under amplitude imbalance. The generalised equations for input impedance of Chireix combiner branches excited by signals with different amplitudes have been derived. The results predicted by the model were validated using both commercial high frequency circuit simulations and experimentally measured results. As a result the proposed model serves two purposes. For one it can be used directly in a larger circuit simulation to predict the performance of an outphasing power amplifier. It can also be used as the basis for a linearization strategy for outphasing power amplifiers.
\end{abstract}

Keywords - behavioral modelling, outphasing amplifier, power amplifiers, nonlinear circuit, Chireix.

\section{INTRODUCTION}

Increasing demand for channel capacity in modern communication systems has led to the use of high-order modulation schemes. Using such modulation schemes in turn requires high transmitter linearity in order to provide low bit error rate. On the other hand, it is desirable that the efficiency of the transmitter should remain as high as possible. In order to fulfil both efficiency and linearity requirements different power amplifier (PA) structures have been proposed [1]-[4]. These PA architectures still cause distortion of the transmitted signal. Not alone due to nonlinear transfer response of the active devices, but also as a result of impedance mismatch and passive structures used. For this reason they are used along with predistortion techniques to ensure linear operation [5].

The concept of an outphasing RF transmitter was initially introduced in [4] and reintroduced in [6] where the author used the term linear amplification with nonlinear components (LINC). The idea of an outphasing PA is to transform amplitude and phase modulated signal into two phase modulated signals using a signal component separator (SCS) as shown in Fig. 1. The two signals coming from the separator have constant envelope which enables them to be amplified with high linearity and efficiency. Ideally, after recombination with a power combiner the output signal should be a replica of the input signal with higher amplitude. However, real outphasing amplifiers will introduce some distortion to the signal. This distortion depends on the branch PAs, the type of the combiner and the parameters of the transmitted signal.

Outphasing amplifiers with isolated, non-isolated and partially isolated combiners have been presented in the literature. However, since the use of an isolating resistor

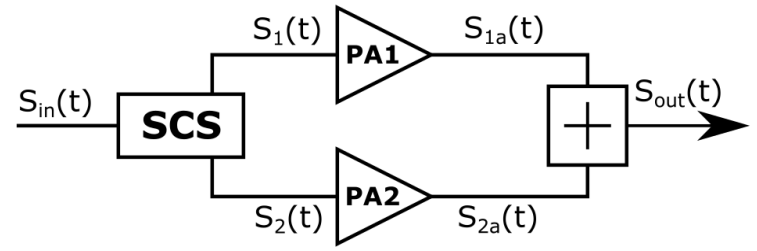

Fig. 1. Block-diagram of a LINC transmitter

degrades the power efficiency [7], non-isolated combiners with compensating reactive elements have more recently found a wider application in outphasing transmitters. Outphasing PAs with non-isolated combiners show significant nonlinear behaviour and phase distortion due to amplitude imbalance between branches [8]. It has been shown that the primary source of this imbalance is load modulation effects in the outphasing PA branches.

Nonlinear properties of non-isolated Chireix combiner have been described in many papers [8]-[11]. However, to date the explicit dependence of nonlinear distortion on amplitude imbalance has not been presented.

In this work we present analytical equations that explicitly attribute how amplitude imbalance between combiner branches distorts the AMAM characteristic of an outphasing amplifier. The proposed analytical equations have been compared to both ADS simulations and experimental measurements.

\section{CHIREIX COMBINER UNDER AMPLITUDE IMBALANCE}

In order to analyse the nonlinear behaviour of a Chireix power combiner, first a basic model of the Chireix combiner is developed as shown in Fig. 2. Here we assume that the branches of the combiner are fed by voltage sources with exciting voltages $\dot{V}_{1}=V_{1} e^{+j \theta}$ and $\dot{V}_{2}=V_{2} e^{-j \theta}$. We denote $k_{V}=V_{1} / V_{2}$ and in the case of amplitude imbalance $k_{V} \neq 1$.

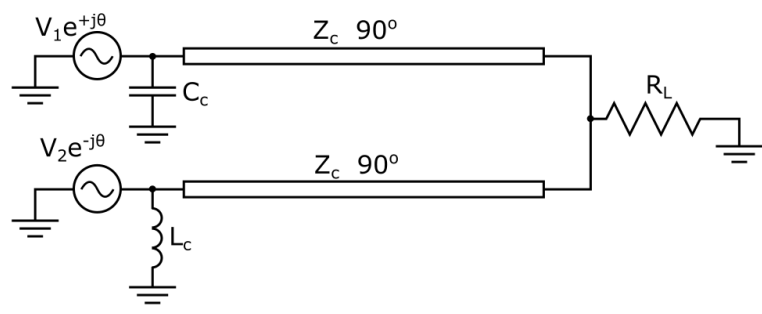

Fig. 2. Circuit model of a Chireix combiner 
Since the sources are connected to the junction point through quarter wavelength transmission lines, the currents next to the load will be $\dot{I}_{1}=I_{1} e^{j \theta}$ and $\dot{I}_{2}=I_{2} e^{-j \theta}$ as shown in Fig. 3. Therefore, the voltage across the load can be expressed as:

$$
\dot{V}_{L}=\left(I_{1} e^{j \theta}+I_{2} e^{-j \theta}\right) R_{L}
$$

and each current source will see impedance:

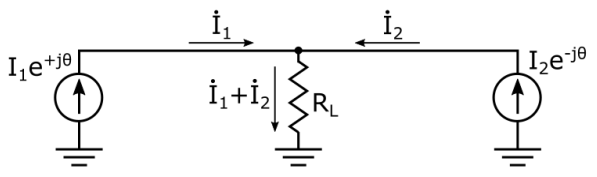

Fig. 3. Currents at the junction

$$
Z_{I 1,2}=\frac{\dot{V}_{L}}{\dot{I}_{1,2}}=R_{L}\left(1+\frac{I_{2,1}}{I_{1,2}} e^{\mp 2 j \theta}\right)
$$

Taking into account that transmission lines of the combiner have characteristic impedance $Z_{c}$ and $k_{V}=V_{1} / V_{2}=I_{1} / I_{2}$ we can express the input impedance of the Chireix combiner transmission lines:

$$
\frac{1}{Z_{1,2}}=\frac{R_{L}}{Z_{c}^{2}}\left(1+\left(k_{V} e^{2 j \theta}\right)^{\mp 1}\right)
$$

If we assume that the compensating reactive elements $C_{c}$ and $L_{c}$ provide the same susceptance $B_{c}=\omega C_{c}=1 /\left(\omega L_{c}\right)$ then the input impedance of the Chireix combiner branches will be:

$$
\frac{1}{Z_{c h 1,2}}=\frac{R_{L}}{Z_{c}^{2}}\left(1+\left(k_{V} e^{2 j \theta}\right)^{\mp 1} \pm j B_{c}\right)
$$

It can be shown that in the particular case when $k_{V}=1$ (4) gives us the well known expressions for input impedance of a Chireix combiner [7].

Having found the input impedance for both branches one can determine the power coming into both branches:

$$
P_{i n 1,2}=\frac{V_{1}^{2}}{2 Z_{1,2}}=\frac{V_{1,2}^{2} R_{L}}{2 Z_{c}^{2}}\left(1+\left(k_{V} e^{2 j \theta}\right)^{\mp 1}\right)
$$

and the power on the load can be found from the energy conservation law:

$$
P_{L}=\operatorname{real}\left\{P_{i n 1}+P_{i n 2}\right\}=\frac{V_{1}^{2} R_{L}}{2 Z_{c}^{2}}\left(1+\frac{1}{k_{V}^{2}}+\frac{2 \cos 2 \theta}{k_{V}}\right)
$$

Therefore amplitude of voltage across the output load can be expressed as:

$$
V_{L}=\sqrt{2 P_{L} R_{L}}=\frac{V_{1} R_{L}}{Z_{c}} \sqrt{1+\frac{1}{k_{V}^{2}}+\frac{2 \cos 2 \theta}{k_{V}}}
$$

From the expression (2) it follows that the phase of the output voltage will be distorted relative to the input voltage by a phase shift, which can be expressed as:

$$
\Delta \theta=\arctan \left(\frac{k_{V}-1}{k_{V}+1} \tan \theta\right)
$$

It should be noted that since the combiner is excited by voltage sources, compensating reactive components $C_{c}$ and $L_{c}$ do not affect the power coming to the load and therefore are not presented in expressions (7) and (8).

\section{CHIREIX COMBINER FED BY POWER SOURCES}

Since all real sources have finite impedance, the proposed technique should be adopted to describe Chireix combiner fed by two power sources with output impedance $r$. In this case each power source will see a parallel connection of transmission lines as the input and compensating reactive element as shown in Fig. 4b.

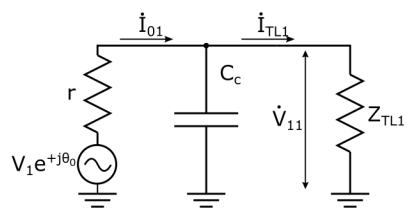

(a)

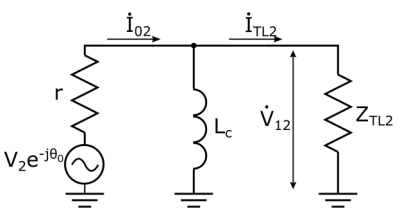

(b)
Fig. 4. Branches of Chireix combiner excited by power sources: (a) - $+\theta$ branch; (b) $--\theta$ branch

In the case of power sources excitation, $r C$ and $r L$ circuits in the combiner branches operate as voltage dividers. Therefore, voltages $\dot{V}_{11}$ and $\dot{V}_{12}$ across inputs $Z_{T L 1}$ and $Z_{T L 2}$ respectively will be different from exciting voltages $\dot{V}_{1}$ and $\dot{V}_{2}$ in both phases and magnitudes. From Ohm's law and equation (3) these voltages can be expressed as:

$$
\begin{gathered}
\dot{V}_{11}=\frac{V_{1} e^{+j \theta} Z_{c}^{2}}{Z_{c}^{2}\left(j r \omega C_{c}+1\right)+r R_{L}\left(1+\frac{1}{k_{V 1}} e^{-j 2 \theta_{1}}\right)} \\
\dot{V}_{12}=\frac{V_{2} e^{-j \theta} Z_{c}^{2} j \omega L_{c}}{Z_{c}^{2}\left(j \omega L_{c}+r\right)+j \omega L_{c} r R_{L}\left(1+k_{V 1} e^{+j 2 \theta_{1}}\right)}
\end{gathered}
$$

where $\omega=2 \pi f_{c}, f_{c}$ is the centre frequency of transmitted signal, $k_{V 1}=V_{11} / V_{12}$ is the amplitude ratio at the input of the transmission lines, $2 \theta_{1}$ is the phase difference between voltages $\dot{V}_{11}$ and $\dot{V}_{12}: 2 \theta_{1}=$ phase $\left(\dot{V}_{11}\right)$-phase $\left(\dot{V}_{12}\right)$. Taking into consideration that $V_{1}=k_{V} V_{2}$ we divide both parts of the equations (9) and (10) and obtain one equation:

$$
\begin{aligned}
& k_{V 1} e^{j \Delta \theta_{1}}=\frac{k_{V} e^{2 j \theta_{0}}}{j \omega L_{c}} \times \\
& \times \frac{Z_{c}^{2}\left(r+j \omega L_{c}\right)+r R_{L} j \omega L_{c}\left(1+k_{V 1} e^{j 2 \theta_{1}}\right)}{Z_{c}^{2}\left(1+j \omega r C_{c}\right)+r R_{L}\left(1+\frac{1}{k_{V 1}} e^{-j 2 \theta_{1}}\right)}
\end{aligned}
$$

Equation (11) can be split into two independent equations for real and imaginary parts. One can exclude $k_{V 1}$ from the system and solve the system for $2 \theta_{1}$. The final result will be:

$$
2 \theta_{1}=\arctan \left(\frac{A_{2} A_{3}-A_{1}}{A_{1} A_{3}+A_{2}}\right)+n \pi
$$

where $A_{1}, A_{2}$ and $A_{3}$ are 


$$
\left\{\begin{array}{l}
A_{1}=K_{1} r R_{L}-Z_{c}^{2}-r R_{L} \\
A_{2}=Z_{c}^{2} r X_{C}-R_{L} r K_{2} \\
A_{3}=\frac{X_{L} r R_{L}-K_{1} X_{L}\left(Z_{c}^{2}+r R_{L}\right)-K_{2} Z_{c}^{2} r}{Z_{c}^{2} X_{L} K_{2}+X_{L} R_{L} r K_{2}-Z_{c}^{2} r K_{1}}
\end{array}\right.
$$

where we denoted $X_{c}=\omega C_{c}, X_{L}=\omega L_{c}, K_{1}=k_{V} \cos 2 \theta_{0}$ and $K_{2}=k_{V} \sin 2 \theta_{0}$. Once the outphasing angle at the input of the Chireix combiner is found, one can find the value of magnitude imbalance at this point:

$$
k_{V 1}=\frac{X_{L} Z_{c}^{2} K_{2}+X_{L} R_{L} r K_{2}-Z_{c}^{2} r K_{1}}{X_{L}\left(D_{1}+D_{2}\right)}
$$

where we denoted:

$$
\left\{\begin{array}{l}
D_{1}=\left(X_{c} Z_{c}^{2} r-r R_{L} K_{2}\right) \cos \left(\Delta \theta_{1}\right) \\
D_{2}=\left(Z_{c}^{2}+r R_{L}-R_{L} r K_{1}\right) \sin \left(\Delta \theta_{1}\right)
\end{array}\right.
$$

Once we have found amplitude and phase imbalance between two combiner branches we can find voltages across the inputs of the combiner transmission lines using the expressions (9) and (10). Amplitude and phase of output voltage can be found in turn from (7) and (8).

\section{VALIDATION OF THE PROPOSED TECHNIQUE}

\section{A. ADS Ptolemy simulation}

In order to validate the nonlinear behaviour of the Chireix combiner excited by two voltage sources we used ADS Ptolemy cosimulation software tool. To test the combiner a 16QAM OFDM modulated signal was used. The original signal had $10 \mathrm{MHz}$ bandwidth and $160 \mathrm{Msps}$ sample rate. This signal was generated using MatLab, separated into two outphasing components and exported to ADS simulation software

In ADS, a Ptolemy cosimulation is carried out by importing the two input signals. They are upconverted in the simulation to a carrier frequency of $3.5 \mathrm{GHz}$ and combined using the Chireix combiner. The output impedance values of the IQ modulators were set to $0.1 \mathrm{Ohm}$. Power imbalance was simulated by additional power added to the $+\theta$ branch $\Delta P=20 \log \left(k_{V}\right)$.

The output signal is then captured using a timed sink block. Having the original signal and the signal received from the output of our testbench, one can plot the AMAM characteristic of the system for different values of $k_{V}$. In Fig. 5 and Fig. 6 we present AMAM curves and phase distortion. The curves simulated using the ADS-Ptolemy cosimulation tool are shown with grey dots and the curves calculated using the proposed model are shown with black dashed lines. The curves are plotted for amplitude imbalance ratios of $k_{V}=1.0,1.2,1.4$, 1.6. For presentation purposes, all the values are normalised to the maximum output voltage in case of linear response. The values of mean square error (MSE) and normalised mean square error (NMSE) are presented in Table 1 as figures of merit for the goodness of fit of the proposed model.

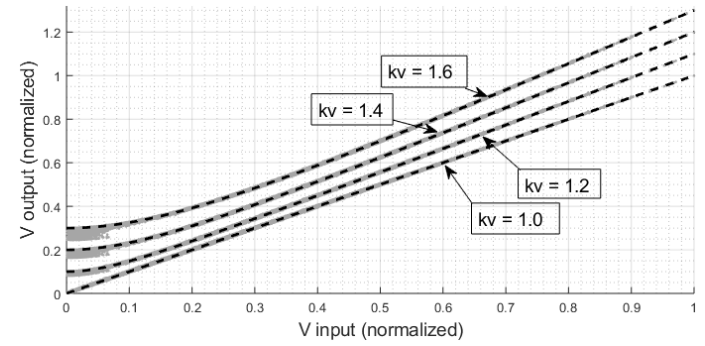

Fig. 5. AMAM characteristics of a Chireix combiner for different values of amplitude imbalance: Simulated in ADS (grey dots); predicted by analytical technique (black dashed line)

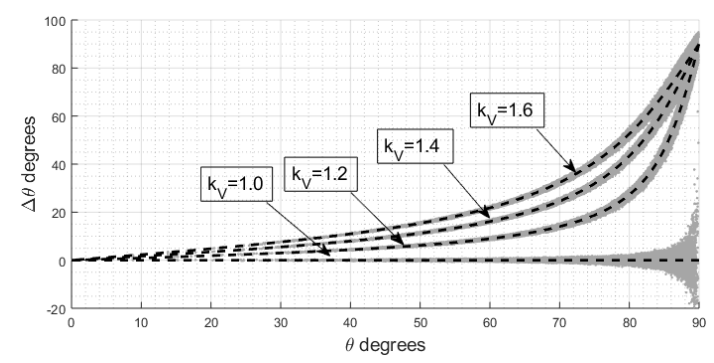

Fig. 6. Phase distortion of a Chireix combiner for different values of amplitude imbalance: Simulated in ADS (grey dots); predicted by analytical technique (black dashed line)

Table 1. MSE and NMSE for different values of amplitude imbalance.

\begin{tabular}{|c|c|c|}
\hline $\mathbf{k}_{\mathbf{V}}$ & MSE, dB & NMSE, dB \\
\hline 1.0 & -42.9 & -34.2 \\
\hline 1.2 & -42.1 & -32.3 \\
\hline 1.4 & -40.7 & -31.7 \\
\hline 1.6 & -39.4 & -31.1 \\
\hline
\end{tabular}

From both the plots and figures of merit, one can see that the results obtained by using the analytical model are in very good agreement with the numerical simulations. However, since the proposed model does not take into account memory effects, the results do not match perfectly.

\section{B. Experimental validation}

For an experimental validation of the proposed technique we used a measurement setup consisting of a dual-path digital-to-analogue converter board and a high-speed analogue-to-digital converter to capture the combined output signal, as shown in Figure 7. An OFDM modulated signal with $10 \mathrm{MHz}$ bandwidth was split into two outphasing paths and generated from the DAC board. The two outphasing parts of the signal are recombined with a microstrip Chireix combiner fabricated on RO4350 substrate. The reactive elements of the combiner are optimised for outphasing angle $73^{\circ}$. The recombined signal was captured with a high-speed analogue-to-digital converter.

Since the outputs of the DAC board have output impedances of $50 \mathrm{Ohm}$, the results are compared with the theoretical results given by equations presented in Section III. It should be noted that unlike the ADS testbench simulation in the experimental setup we reduced the power of the $-\theta$ branch. 


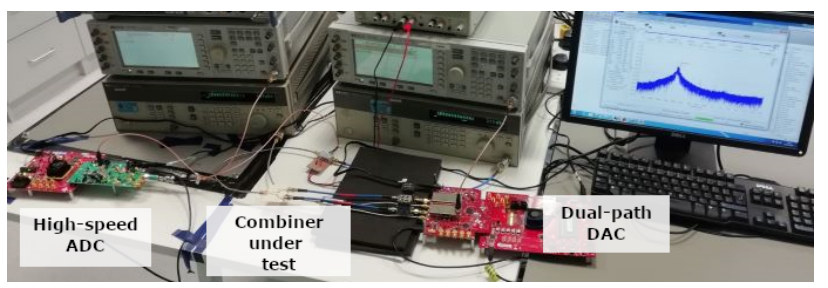

Fig. 7. Experimental setup

For this reason the bigger the value of $k_{V}$ the less power that will come to the output load. The MSE and NMSE values for the model compared to the experimentally measured complex envelope responses are listed in Table 2. The AMAM distortion of the signal for different values of magnitude imbalance $k_{V}$ is presented in Figure 8.

Table 2. MSE and NMSE for different values of amplitude imbalance.

\begin{tabular}{|c|c|c|}
\hline $\mathbf{k}_{\mathbf{V}}$ & MSE, dB & NMSE, dB \\
\hline 1.0 & -37.19 & -31.25 \\
\hline 1.2 & -35.2 & -29.38 \\
\hline 1.4 & -33.67 & -28.12 \\
\hline 1.6 & -32.16 & -26.88 \\
\hline
\end{tabular}

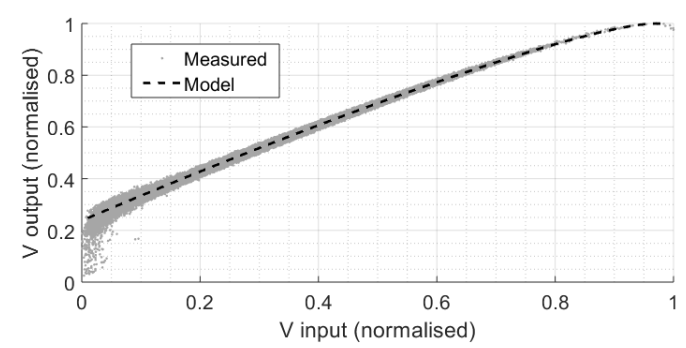

(a)

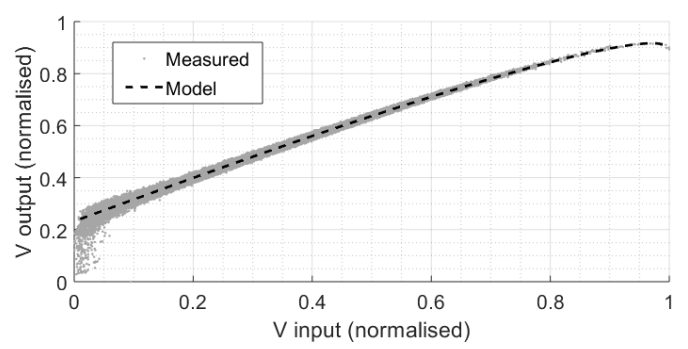

(b)

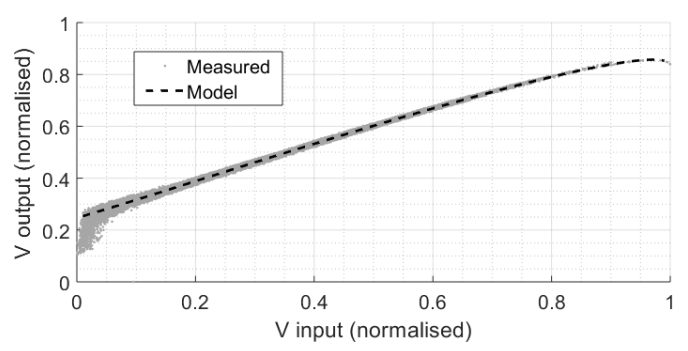

(c)

Fig. 8. Measured AMAM characteristics for Chireix combiner excited by two power sources for different values of $k_{V}$ : (a) $k_{V}=1.0$; (b) $k_{V}=1.2$; (c) $k_{V}=1.4$
Comparing Figures 5 and 8 reveals that nonlinearity in the case of voltage source and power source are completely different. When the combiner shoulders are excited with voltage source, amplitude imbalance is the only source of nonlinearity, whereas when they are fed with power sources nonlinear distortion is also caused by the $r C$ and $r L$ voltage dividers. From these results it follows that, to study nonlinear behaviour of outphasing amplifiers the behaviour of branch PAs must be taken into account.

\section{CONCLUSION}

A new analysis of Chireix power combiner has been presented that is used to derive a model which can describe the effect of amplitude imbalance between two branches. Explicit analytical expressions for output voltage have been derived. The derived model is validated using simulations in commercial high frequency simulator and experimental measurements. Plots and figures of merit for goodness of fit have been provided for a range of amplitude imbalance levels and show good agreement with the experimentally measured performance. Future work will include introduction memory effect in the model.

\section{ACKNOWLEDGMENT}

This publication has emanated from research conducted with the financial support of Science Foundation Ireland (SFI) and is co-funded under the European Regional Development Fund under Grant Number 13/RC/2077.

\section{REFERENCES}

[1] W. H. Doherty, "A new high efficiency power amplifier for modulated waves," Proceedings of the Institute of Radio Engineers, vol. 24, no. 9, pp. 1163-1182, Sept 1936.

[2] F. H. Raab, P. Asbeck, S. Cripps, P. B. Kenington, Z. B. Popovic, N. Pothecary, J. F. Sevic, and N. O. Sokal, "Power amplifiers and transmitters for RF and microwave," IEEE Trans. Microw. Theory Techn., vol. 50, no. 3, pp. 814-826, March 2002.

[3] L. R. Kahn, "Single-sideband transmission by envelope elimination and restoration," Proceedings of the IRE, vol. 40, no. 7, pp. 803-806, July 1952.

[4] H. Chireix, "High power outphasing modulation," Proceedings of the Institute of Radio Engineers, vol. 23, no. 11, pp. 1370-1392, Nov 1935.

[5] F. M. Ghannouchi and O. Hammi, "Behavioral modeling and predistortion," IEEE Microw. Mag., vol. 10, no. 7, pp. 52-64, Dec 2009.

[6] D. Cox, "Linear amplification with nonlinear components," IEEE Trans. Commun., vol. 22, no. 12, pp. 1942-1945, December 1974.

[7] F. Raab, "Efficiency of outphasing RF power-amplifier systems," IEEE Trans. Commun., vol. 33, no. 10, pp. 1094-1099, October 1985.

[8] A. Birafane and A. B. Kouki, "On the linearity and efficiency of outphasing microwave amplifiers," IEEE Trans. Microw. Theory Techn., vol. 52, no. 7, pp. 1702-1708, July 2004.

[9] S. Boumaiza and F. M. Ghannouchi, "Study and minimization of the outphasing amplifiers nonlinearity," in 2006 European Microwave Conference, Sept 2006, pp. 188-191.

[10] T. Hwang, K. Azadet, R. S. Wilson, and J. Lin, "Nonlinearity modeling of a chireix outphasing power amplifier," IEEE Trans. Circuits Syst. I, vol. 62 , no. 12 , pp. 2898-2907, Dec 2015.

[11] A. Koukab and O. T. Amiri, "Circuit-oriented approach for modeling and analysis of outphasing transmitters," in 2012 International Symposium on Communications and Information Technologies (ISCIT), Oct 2012, pp. 234-238. 